\title{
AN EXTENSION OF THE HAUSDORFF-TOEPLITZ THEOREM ON THE NUMERICAL RANGE
}

\author{
YIK-HOI AU-YEUNG AND NAM-KIU TSING
}

\begin{abstract}
Let $H_{n}$ be the set of all $n \times n$ hermitian matrices and $U_{n}$ the set of all $n \times n$ unitary matrices. For any $c=\left(c_{1}, \ldots, c_{n}\right) \in \mathbf{R}^{n}$ and $A_{1}, A_{2}, A_{3} \in \mathscr{H}_{n}$, let $W(A, A:, A:)$ denote the set

$$
\left\{\left(\operatorname{tr}[c] U A_{1} U^{*}, \operatorname{tr}[c] U A_{2} U^{*}, \operatorname{tr}[c] U A_{3} U^{*}\right): U \in U_{n}\right\},
$$

where $[c]$ is the diagonal matrix with $c_{1}, \ldots, c_{n}$ as diagonal entries. In this present note, the authors prove that if $n>2$, then $W_{c}\left(A_{1}, A_{2}, A_{3}\right)$ is always convex. Equivalent statements of this result, in terms of definiteness and inclusion relations, are also given. These results extend the theorems of Hausdorff-Toeplitz, Finsler and Westwick on numerical ranges, respectively.
\end{abstract}

1. Introduction. Let $\mathscr{H}_{n}$ be the set of all $n \times n$ (complex) hermitian matrices and $U_{n}$ the set of all $n \times n$ unitary matrices. For any $c=\left(c_{1}, \ldots, c_{n}\right) \in \mathbf{R}^{n}$ and $A_{1}, \ldots, A_{p} \in \mathcal{H}_{n}$, let $W_{c}\left(A_{1}, \ldots, A_{p}\right)=\left\{\left(\operatorname{tr}[c] U A_{1} U^{*}, \ldots, \operatorname{tr}[c] U A_{p} U^{*}\right): U \in \mathcal{U}_{n}\right\}$, where ' $\operatorname{tr}$ ' means the trace and $[c]$ is the diagonal matrix with $c_{1}, \ldots, c_{n}$ as diagonal entries. In the 1910's Toeplitz and Hausdorff $[12,10]$ proved (in an equivalent form) that $W_{c}\left(A_{1}, A_{2}\right)$ is convex for $c=(1,0, \ldots, 0)$. In the 1960's Halmos [9] conjectured and Berger [3] proved that if $k$ coordinates of $c$ are 1 and the rest are 0 , then $W_{c}\left(A_{1}, A_{2}\right)$ is convex. In the 1970 's, it was Westwick [13] who proved that $W_{c}\left(A_{1}, A_{2}\right)$ is convex for any $c \in \mathbf{R}^{n}$ (for another proof see Poon [11]). By using a result of Bohnenblust [4] (see Friedland and Loewy [7] for another proof) it is not difficult to see (for example, see [1]) that $W_{c}\left(A_{1}, \ldots, A_{p}\right)$ is convex for $c=$ $(1,0, \ldots, 0), p=3$ and $n>2$ and this result is best possible in the sense that the result fails to hold if $p>3$ or $n=2$. As from Toeplitz- Hausdorff to Westwick it is quite natural to ask whether $W_{c}\left(A_{1}, A_{2}, A_{3}\right)$ is convex for $n>2$ and for any $c \in \mathbf{R}^{n}$. In this note, we shall give an affirmative answer to this question.

2. Convexity of $W_{c}\left(A_{1}, A_{2}, A_{3}\right)$. For any $b, c \in \mathbf{R}^{n}$, we write $b \ll c$ if there is a doubly-stochastic matrix $D$ (i.e. $D$ is nonnegative and all row sums and column sums are equal to 1 ) such that $b=c D$. The proof of the following lemma is similar to that of Theorem 1 in [2], although there we consider only the case $p=2$.

Lemma 1. For any $c=\left(c_{1}, \ldots, c_{n}\right) \in \mathbf{R}^{n}$ and for any $A_{1}, \ldots, A_{p} \in \mathcal{H}_{n}$, the following conditions are equivalent:

(i) $W_{c}\left(A_{1}, \ldots, A_{p}\right)$ is convex.

(ii) If $\left(r_{1}, \ldots, r_{p}\right) \in \mathbf{R}^{p} \backslash W_{c}\left(A_{1}, \ldots, A_{p}\right)$, then there exist $\alpha_{1}, \ldots, \alpha_{p} \in \mathbf{R}$ such that

$$
\operatorname{tr}\left([c] U\left(\sum_{j=1}^{p} \alpha_{j} A_{j}\right) U^{*}\right)>\sum_{j=1}^{p} \alpha_{j} r_{j}, \quad \text { for all } U \in \mathcal{U}_{n} .
$$

Received by the editors November 23, 1982.

1980 Mathematics Subject Classification. Primary 47A12; Secondary 15A60.

Key words and phrases. Numerical range, convexity, doubly-stochastic matrix, convex hull. 
(iii) If $\left(r_{1}, \ldots, r_{p}\right) \in \mathbf{R}^{p} \backslash W_{c}\left(A_{1}, \ldots, A_{p}\right)$, then there exist $\alpha_{1}, \ldots, \alpha_{p} \in \mathbf{R}$ such that

$$
\sum_{i=1}^{n} c_{i} \lambda_{\sigma(i)}>\sum_{j=1}^{p} \alpha_{j} r_{j}
$$

for all $\sigma \in S_{n}$, where $\lambda_{1}, \ldots, \lambda_{n}$ are the eigenvalues of $\sum_{j=1}^{p} \alpha_{j} A_{j}$ and $S_{n}$ is the permutation group of order $n$.

(iv) $W_{b}\left(A_{1}, \ldots, A_{p}\right) \subset W_{c}\left(A_{1}, \ldots, A_{p}\right)$ for all $b \ll c$.

An $n \times n$ doubly-stochastic matrix $Q=\left(q_{i j}\right)$ is called a pinching matrix if for some $1 \leq k<l \leq n$ and $0 \leq \mu \leq 1$,

$$
q_{i j}= \begin{cases}\mu, & \text { if }(i, j)=(k, k) \text { or }(l, l), \\ 1-\mu, & \text { if }(i, j)=(k, l) \text { or }(l, k), \\ 1, & \text { if } i=j \neq k, l, \\ 0, & \text { otherwise }\end{cases}
$$

The following lemma is well known (for example, see [8]).

LEMMA 2. For any $b, c \in \mathbf{R}^{n}$, we have $b \ll c$ if and only if $b=c Q$ where $Q$ is a finite product of pinching matrices.

The idea used in the proof of the following theorem has been used in [2].

Theorem. If $c \in \mathbf{R}^{n}, A_{1}, A_{2}, A_{3} \in \mathcal{H}_{n}$ and $n>2$, then the following conditions hold:

(i) $W_{c}\left(A_{1}, A_{2}, A_{3}\right)$ is convex.

(ii) If $\left(r_{1}, r_{2}, r_{3}\right) \in \mathbf{R}^{3} \backslash W_{c}\left(A_{1}, A_{2}, A_{3}\right)$, then there exist $\alpha_{1}, \alpha_{2}, \alpha_{3} \in \mathbf{R}$ such that

$$
\operatorname{tr}\left([c] U\left(\sum_{j=1}^{3} \alpha_{j} A_{j}\right) U^{*}\right)>\sum_{j=1}^{3} \alpha_{j} r_{j}
$$

for all $U \in U_{n}$.

(iii) If $\left(r_{1}, r_{2}, r_{3}\right) \in \mathbf{R}^{3} \backslash W_{c}\left(A_{1}, A_{2}, A_{3}\right)$, then there exist $\alpha_{1}, \alpha_{2}, \alpha_{3} \in \mathbf{R}$ such that

$$
\sum_{i=1}^{n} c_{i} \lambda_{\sigma(i)}>\sum_{j=1}^{3} \alpha_{j} r_{j}
$$

for all $\sigma \in S_{n}$, where $\lambda_{1}, \ldots, \lambda_{n}$ are the eigenvalues of $\sum_{j=1}^{3} \alpha_{j} A_{j}$ and $S_{n}$ is the permutation group of order $n$.

(iv) $W_{b}\left(A_{1}, A_{2}, A_{3}\right) \subset W_{c}\left(A_{1}, A_{2}, A_{3}\right)$ for all $b \ll c$.

Proof. By Lemmas 1 and 2, it suffices to prove that

$$
W_{b}\left(A_{1}, A_{2}, A_{3}\right) \subset W_{c}\left(A_{1}, A_{2}, A_{3}\right),
$$

where

$$
\left\{\begin{array}{l}
b_{k}=\mu c_{k}+(1-\mu) c_{l}, \\
b_{l}=(1-\mu) c_{k}+\mu c_{l}, \\
b_{i}=c_{i} \text { for all } i \neq k, l
\end{array}\right.
$$

for some $1 \leq k<l \leq n$ and $0 \leq \mu \leq 1$. We may also assume, without loss of generality, that $(k, l)=(1,2)$. Let $\Lambda_{n}$ denote the set of all (ordered) orthonormal 
bases in $\mathbf{C}^{n}$. Then $W_{c}\left(A_{1}, A_{2}, A_{3}\right)$ is equal to the set

$$
\left\{\left(\sum_{i=1}^{n} c_{i} x_{i} A_{1} x_{i}^{*}, \sum_{i=1}^{n} c_{i} x_{i} A_{2} x_{i}^{*}, \sum_{i=1}^{n} c_{i} x_{i} A_{3} x_{i}^{*}\right):\left(x_{1}, \ldots, x_{n}\right) \in \Lambda_{n}\right\} .
$$

Let $\left(r_{1}, r_{2}, r_{3}\right) \in W_{b}\left(A_{1}, A_{2}, A_{3}\right)$. Then there is $\left(e_{1}, \ldots, e_{n}\right) \in \Lambda_{n}$ such that

$$
\begin{aligned}
r_{j} & =\sum_{i=1}^{n} b_{i} e_{i} A_{j} e_{i}^{*} \\
& =\frac{1}{2}\left(b_{1}+b_{2}\right)\left(e_{1} A_{j} e_{1}^{*}+e_{2} A_{j} e_{2}^{*}\right)+\frac{1}{2}\left(b_{1}-b_{2}\right)\left(e_{1} A_{j} e_{1}^{*}-e_{2} A_{j} e_{2}^{*}\right)+\sum_{i=3}^{n} b_{i} e_{i} A_{j} e_{i}^{*}
\end{aligned}
$$

for $j=1,2,3$. For any $\theta, \phi \in \mathbf{R}$ and $\left(x_{1}, \ldots, x_{n}\right) \in \Lambda_{n}$, define $y_{1}=\cos \theta x_{1}+$ $\sin \theta e^{\phi \sqrt{-1}} x_{2}, y_{2}=-\sin \theta x_{1}+\cos \theta e^{\phi \sqrt{-1}} x_{2}$ and $y_{i}=x_{i}$ for all $i \geq 3$. Then $\left(y_{1}, \ldots, y_{n}\right) \in \Lambda_{n}$ and for $j=1,2,3$,

$$
\begin{aligned}
\sum_{i=1}^{n} c_{i} y_{i} A_{j} y_{i}^{*}= & \frac{1}{2}\left(c_{1}+c_{2}\right)\left(x_{1} A_{j} x_{1}^{*}+x_{2} A_{j} x_{2}^{*}\right) \\
& +\frac{1}{2}\left(c_{1}-c_{2}\right)\left[p_{j} \cos 2 \theta+\sin 2 \theta\left(q_{j} \cos \phi+s_{j} \sin \phi\right)\right] \\
& +\sum_{i=3}^{n} c_{i} x_{i} A_{j} x_{i}^{*}
\end{aligned}
$$

where

$$
p_{j}=x_{1} A_{j} x_{1}^{*}-x_{2} A_{j} x_{2}^{*}, \quad q_{j}=2 \operatorname{Re}\left(x_{1} A_{j} x_{2}^{*}\right), \quad s_{j}=2 \operatorname{Im}\left(x_{1} A_{j} x_{2}^{*}\right)
$$

are real scalars. As $\theta$ and $\phi$ vary in $\mathbf{R}$, the locus of the point

$$
\left(\sum_{i=1}^{n} c_{i} y_{i} A_{1} y_{i}^{*}, \sum_{i=1}^{n} c_{i} y_{i} A_{2} y_{i}^{*}, \sum_{i=1}^{n} c_{i} y_{i} A_{3} y_{i}^{*}\right)
$$

in $\mathbf{R}^{3}$ is an ellipsoid, which we shall denote by $E_{\left(x_{1}, \ldots, x_{n}\right)}$ and is degenerate (hence convex) if $c_{1}=c_{2}$ or

$$
\operatorname{det}\left(\begin{array}{lll}
p_{1} & q_{1} & s_{1} \\
p_{2} & q_{2} & s_{2} \\
p_{3} & q_{3} & s_{3}
\end{array}\right)=0
$$

It is clear that $E_{\left(x_{1}, \ldots, x_{n}\right)} \subset W_{c}\left(A_{1}, A_{2}, A_{3}\right)$ for any $\left(x_{1}, \ldots, x_{n}\right) \in \Lambda_{n}$. Since $c_{1}+c_{2}=$ $b_{1}+b_{2},\left|c_{1}-c_{2}\right| \geq\left|b_{1}-b_{2}\right|$ and $c_{i}=b_{i}$ for all $i \geq 3,\left(r_{1}, r_{2}, r_{3}\right) \in \operatorname{conv} E_{\left(e_{1}, \ldots, e_{n}\right)}$ where "conv" denotes the convex hull. If $\left|c_{1}-c_{2}\right|=\left|b_{1}-b_{2}\right|$ or $E_{\left(e_{1}, \ldots, e_{n}\right)}$ degenerates, then $\left(r_{1}, r_{2}, r_{3}\right) \in E_{\left(e_{1}, \ldots, e_{n}\right)} \subset W_{c}\left(A_{1}, A_{2}, A_{3}\right)$. So we may assume that $E_{\left(e_{1}, \ldots, e_{n}\right)}$ does not degenerate and $\left(r_{1}, r_{2}, r_{3}\right)$ lies in the interior of the ellipsoid $E_{\left(e_{1}, \ldots, e_{n}\right)}$. Let $w_{1} \in \mathbf{C}^{n}$ be a unit eigenvector of $A_{1}$. Since $n>2$, we can choose a unit vector $w_{2} \in \mathbf{C}^{n}$ which is orthogonal to both $w_{1}$ and $w_{1} A_{2}$. Consider a continuous function $f:[0,1] \rightarrow \Lambda_{n}$ such that $f(0)=\left(e_{1}, \ldots, e_{n}\right)$ and $f(1)=\left(w_{1}, \ldots, w_{n}\right)$, where $\left(w_{1}, \ldots, w_{n}\right)$ is any extension of $\left(w_{1}, w_{2}\right)$ to an orthonormal basis of $\mathbf{C}^{n}$. Now $\left(r_{1}, r_{2}, r_{3}\right)$ lies within the interior of $E_{f(0)}$, and $E_{f(1)}$ degenerates (hence convex) as $w_{1} A_{j} w_{2}^{*}=0$ for $j=1,2$. If $\left(r_{1}, r_{2}, r_{3}\right) \in E$, then $\left(r_{1}, r_{2}, r_{3}\right)$ is automatically in $W_{c}\left(A_{1}, A_{2}, A_{3}\right)$. If $\left(r_{1}, r_{2}, r_{3}\right) \notin E_{f(1)}$, then, by continuity, there exists $\alpha \in(0,1)$ such that $\left(r_{1}, r_{2}, r_{3}\right) \in E_{f(\alpha)}$. Thus $\left(r_{1}, r_{2}, r_{3}\right) \in W_{c}\left(A_{1}, A_{2}, A_{3}\right)$.

By taking $c=(1,0, \ldots, 0)$ in (iii) of the theorem, we get the counterpart of a result of Finsler [6] (for another proof of Finsler's result see Calabi [5]). 
Corollary. If $A_{1}, A_{2}, A_{3} \in H_{n}$, where $n>2$, are such that $\sum_{j=1}^{3}\left(x A_{j} x^{*}\right)^{2} \neq 0$ for all nonzero $x \in \mathbf{C}^{n}$, then there exist $\alpha_{1}, \alpha_{2}, \alpha_{3} \in \mathbf{R}$ such that $\sum_{j=1}^{3} \alpha_{j} A_{j}$ is positive definite.

REMARK. Statements (i) and (iv) of the theorem are generalizations of results of Westwick [13] and Goldberg-Straus [8], respectively, for $n>2$.

\section{REFERENCES}

1. Y. H. Au-Yeung and Y. T. Poon, A remark on the convexity and positive definiteness concerning hermitian matrices, Southeast Asian Bull. Math. 3 (1979), 85-92.

2. Y. H. Au-Yeung and N. K. Tsing, Some theorems on the generalized numerical ranges, Linear and Multilinear Algebra (to appear).

3. C. A. Berger, Normal dilations, Cornell Univ., Doctoral dissertation, 1963.

4. F. Bohnenblust, Joint positiveness of matrices, unpublished manuscript.

5. E. Calabi, Linear systems of real quadratic forms, Proc. Amer. Math. Soc. 15 (1964), 844-846.

6. P. Finsler, Über das Vorkommen definiter und semidefiniter Formen in Scharen quadratischer - Formen, Comment. Math. Helv. 9 (1936/37), 188-192.

7. S. Friedland and R. Loewy, Subspaces of symmetric matrices containing matrices with a multiple first eigenvalue, Pacific J. Math. 62 (1976), 389-399.

8. M. Goldberg and E. G. Straus, Elementary inclusion relations for generalized numerical ranges, Linear Algebra Appl. 18 (1977), 1-24.

9. P. Halmos, A Hilbert space problem book, Von Nostrand, New York, 1967.

10. F. Hausdorff, Der Wertvorrat einer Bilinearform, Math. Z. 3 (1919), 314-316.

11. Y. T. Poon, Another proof of a result of Westurick, Linear and Multilinear Algebra 9 (1980), 35-37.

12. O. Toeplitz, Das algebraishe Analogon zu einum Satze von Fejér, Math. Z. 2 (1918), 187-197.

13. R. Westwick, $A$ theorem on numerical ranges, Linear and Multilinear Algebra 2 (1975), 311315 .

Department of Mathematics, University of Hong Kong, Hong Kong 